\title{
Generalized partition functions and interpolating statistics 因
}

\author{
P. F. Borgest H. Boschi-Filho ${ }^{\dagger \ddagger}$ and C. Farina ${ }^{\dagger}$ \\ ${ }^{\dagger}$ Instituto de Física, Universidade Federal do Rio de Janeiro \\ Cidade Universitária, Ilha do Fundão, Caixa Postal 68528 \\ 21945-970 Rio de Janeiro, BRAZIL \\ and \\ $¥$ Center for Theoretical Physics \\ Laboratory for Nuclear Science \\ Massachusetts Institute of Technology \\ Cambridge, Massachusetts 02139-430\%, USA
}

July 6, 2021

\begin{abstract}
We show that the assumption of quasiperiodic boundary conditions (those that interpolate continuously periodic and antiperiodic conditions) in order to compute partition functions of relativistic particles in $2+1$ space-time can be related with anyonic physics. In particular, in the low temperature limit, our result leads to the well known second virial coefficient for anyons. Besides, we also obtain the high temperature limit as well as the full temperature dependence of this coefficient.
\end{abstract}

${ }^{*}$ This work is supported in part by funds provided by the U.S. Department of Energy (D.O.E.) under cooperative research agreement \#DF-FC02-94ER40818 and Conselho Nacional de Desenvolvimento Científico e Tecnológico (CNPq) - Brazilian agency.

†E-mail addresses: pborges@if.ufrj.br; boschi@ctp.mit.edu; and farina@if.ufrj.br. 
In nature one encounters particles which obey Bose-Einstein (BE) or Fermi-Dirac (FD) statistics. However, speculations concerning the existence of particles which obey intermediate (fractional) statistics between BE and FD have been raised for a long time. In recent years this kind of conjecture has gained force with its possible relation with condensed matter effects like high- $T_{C}$ superconductivity and the fractional quantum Hall effect [1]].

In $(2+1)$ dimensional field theory, fractional statistics can be obtained by including a Chern-Simons term [2] in the Lagrangian density describing the interaction of matter with gauge fields. Particles with intermediate statistics between the BE and FD cases in two space dimensions are usually called anyons. Their properties can be understood from the braid group or equivalently assuming that they are hard core indistinguishable particles in two space dimensions so that the configuration space becomes a manifold $\mathcal{M}$ with a non-trivial topology in the sense that $\Pi_{1}(\mathcal{M})=B_{N}$ for $N$ particles, where $B_{N}$ denotes the braid group with $N$ generators. Hence, it can be shown that when anyons turn around each other the corresponding wave function (or the Feynman propagator for the system) acquires a non-trivial geometric phase [3]-[6]. An inherent difficulty of the anyon field description is its non-locality [7]. This makes it difficult to calculate beyond the second virial coefficient [8] even in the case of "free" anyons, contrary to what happens for free bosonic and fermionic gases for which the full partition function is known.

Here, we shall discuss a different approach to the interpolation between bosons and fermions. In this paper we shall compute a generalized partition function which contains the relativistic partition functions for bosons and fermions as particular cases, and also interpolates continuously between these cases. From this partition function we obtain the second virial coefficient and show that, in the low energy (temperature) limit it reproduces correctly the results known for anyons in the literature [8]. We also find the high temperature and the full temperature dependence of the relativistic second virial coefficient.

Let us briefly review how to obtain the partition function for ideal relativistic bosonic and fermionic gases, from which we will generalize to the anyonic case. The partition function for a system described by a Hamiltonian $H$ with chemical potential $\mu$ can be written as

$$
\mathcal{Z} \equiv \exp \{-\beta \Omega\}=\operatorname{Tr} e^{-\beta(H+\mathcal{N} \mu)},
$$

where $\Omega$ is the free energy and $\mathcal{N}$ is the conserved charge of the system. As it is well known for relativistic charged massive bosonic fields one can express this partition function as a determinant 90], namely,

$$
\mathcal{Z}=\left[\left.\operatorname{det}\left(-D^{2}+M^{2}\right)\right|_{P}\right]^{-1} ; \quad \text { (bosons) }
$$

where $D^{2}$ is the square of the covariant derivative, $D_{\nu}$, including the chemical potential, $D_{\nu}=\left(\partial_{0}+i \mu, \partial_{i}\right)$ and $M$ is the mass of the field. This prescription for the inclusion of the 
chemical potential $\mu$ coincides with the one given by eq. (11) except for the introduction of an imaginary part to $\mathcal{Z}$, which is not physically relevant. So unless otherwise specified, we are considering that the real part of $\mathcal{Z}$ has been taken. Note also that, the chemical potential appears squared in the relevant operator $-D^{2}+M^{2}$. This is a consequence of representing the partition function in phase space and then integrating over momenta (see 110 for details). The label $P$ means that the eigenvalues of this operator are subjected to periodic boundary conditions and hence are given by

$$
\lambda_{n k}=\left(\omega_{n}+i \mu\right)^{2}+\vec{k}^{2}+M^{2},
$$

where $\omega_{n}=2 n \pi / \beta$, with $n \in \mathbb{Z}$, are the Matsubara frequencies [11] for bosonic fields and $\vec{k} \in \mathbb{R}^{N}$.

As for the case of bosons, one can also write the partition function for free relativistic fermions as a determinant

$$
\begin{aligned}
\mathcal{Z} & =\left.\operatorname{det}_{D}(\not D D-M)\right|_{A} \\
& =\left[\left.\operatorname{det}\left(-D^{2}+M^{2}\right)\right|_{A}\right]^{d / 2} ; \quad \text { (fermions), }
\end{aligned}
$$

where the determinants are calculated over the corresponding operator and $\operatorname{det}_{D}$ means the inclusion of the calculation over Dirac indices ( $d$ is the dimension of the Dirac representation which hereafter we take $d=2$ ). The subscript $A$ means that the eigenvalues of $-D^{2}+M^{2}$ are now computed with antiperiodic boundary conditions. Hence, they are given by (3), but now the Matsubara frequencies are $\omega_{n}=(2 \pi / \beta)(n+1 / 2)$, due to the antiperiodic boundary condition.

The remarkable fact about the (last line of) equation (田) is the well known but few explored character that it states that it is possible to compute the partition function for fermions through an equation where spin does not appear at all. The information that the partition function (4) corresponds to fermions is solely described by the fact that the determinant of the Klein-Gordon operator $\left(-D^{2}+M^{2}\right)$ is computed over antiperiodic eigenfunctions. Naturally, this antiperiodicity comes from the fact that fermions anticommute when they are exchanged in space, i. e., the wave function of the system acquires a sign $(-1)$. So, on thermodynamical grounds the only necessary information to compute a partition function for free fermions is that they obey energy conservation (expressed through the Klein-Gordon operator) and that their wave functions are antiperiodic in the Euclidean time.

Now, we want to extend this fundamental result to the case of (quasi) particles that obey an intermediate statistics between the bosonic and fermionic cases. As is well known these particles live in $2+1$ dimensional space-time and they can be described by specific equations of motion like the one of Jackiw and Nair [12]. This equation of motion governs 
the dynamics of that particles and contains detailed information on their algebraic properties, including the braid group as well. However, the task of computing a determinant of an operator obtained from such equation is very involved and we are not going in this direction in this work.

An alternate route, that we are going to follow here, is to use very few but essential information which are necessary for a thermodynamical description of a system of such particles. These informations are only three: first these (relativistic) particles should obey energy conservation and so in spite that they are described by some involved specific $2+1$ dimensional equation they should also obey the Klein-Gordon equation; second the determinant of the Klein-Gordon operator should be calculated using some appropriate boundary condition; Finally the partition function is defined by a certain power $\sigma$ (to be discussed later) of the determinant of the Klein-Gordon operator, under a chosen boundary condition. Of course, if the boundary condition is periodic in Euclidean time and $\sigma=-1$ we will be describing bosons as is expressed in equation (2). On the other hand, if we impose antiperiodic boundary conditions and $\sigma=+1$ we will be describing fermions as in equation (四). As we want to describe particles that obey some statistics that continuously interpolate the bosonic and fermionic cases, it seems rather natural to choose a boundary condition that interpolates these two well known cases. Such a condition, which we call quasiperiodic, can be written as

$$
\psi(0 ; \vec{x})=e^{i \theta} \psi(\beta ; \vec{x})
$$

where $\theta$ is a continuous parameter defined in the interval $[0, \pi]$. Note that putting $\theta=0$ we reduce the above condition to a periodic boundary condition and if $\theta=\pi$ it becomes an antiperiodic one.

The power $\sigma$ of the determinant is introduced to fit the bosonic and fermionic cases. As is well known in these cases the powers -1 and +1 , respectively, come from the definition of the measure over c-number (bosons) and Grasman variables (fermions). For the anyonic case it would be necessary to define a set of variables which could interpolate the properties of c-numbers and Grasman variables. This approach seems to be related to q-deformed calculations, but here we are just going to leave the power $\sigma$ of the determinant free and check, at the end of the calculations that both $\sigma= \pm 1$ will be related to anyonic physics. This may be explained remembering that conventional anyons are constructed from fermions or bosons (with a fixed built in measure) adding a Chern-Simons term. Later on, we will also show that keeping $\theta$ fixed and varying $\sigma$ we could describe as well the interpolation between bosons and fermions.

Note that the quasiperiodic condition (5) can also follow from an analogy of the spatial behavior of bosons, fermions and anyons. This behavior is the well known property that bosonic wave functions are symmetrical while fermionic ones are antisymmetrical and anyonic wave functions acquire a phase $\exp (i \theta)$ when these particles are turned around 
each other [1, 13]. Going to the finite temperature case, these properties imply that bosonic fields are periodic in Euclidean time while fermionic are antiperiodic. This analogy is not complete for anyons and we are assuming here that an intermediate continuous condition can explain at least some of the thermodynamical properties of anyons, as we are going to show bellow.

Then, we are not proving that anyons obey the quasiperiodic boundary condition, eq. (5), and we are not able at this point to do so, but we are using a very appealing analogy to support it. The condition (5) is our basic assumption from which we are going to show that the partition function that we will calculate can be mapped onto the corresponding results for conventional anyons. In fact we do this for the only exact result known for anyons in this matter which is the second virial coefficient obtained by Arovas et. al. [8]. The calculation of higher virial coefficients and its comparison with the perturbative or numerical ones known in the literature is done in a forthcoming work [14].

The price we have to pay in our approach is that we are loosing detailed quantum mechanical information as those associated with the braid group which become very involved when many particles come into play. The obvious advantage of this global approach is that it keeps only the essential thermodynamical information which suffices for calculating the virial coefficients, for example.

Note also that, in our approach there is no reason for restricting the space-time dimensions, despite that anyons live only in two space dimensions. This is not surprising since other approaches towards generalized statistics, like the Haldane's generalized exclusion principle [15], can be formulated in spaces with arbitrary number of dimensions. In fact, at the end of our calculations we shall restrict them to two space dimensions in order to show the equivalence of our approach with the conventional one for anyons.

Let us now compute the following determinant of the Klein-Gordon operator:

$$
\mathcal{Z}=\left[\left.\operatorname{det}\left(-D^{2}+M^{2}\right)\right|_{\theta}\right]^{\sigma}
$$

where the subscript $\theta$ means that the quasiperiodic condition (5) is assumed and we introduced the parameter $\sigma$ to be able to reproduce correctly the bosonic and fermionic particular cases. Observe that when $\theta=0$ and $\sigma=-1$ we reobtain the bosonic partition function, while for $\theta=\pi$ and $\sigma=+1$ we have the fermionic case. For generality, we are going to calculate the above determinant in $N+1$ space-time dimensions. The eigenvalues of the Klein-Gordon operator in this case are:

$$
\lambda_{n k \theta}=\left[\frac{2 n \pi}{\beta}+i \mu+\frac{\theta}{\beta}\right]^{2}+\vec{k}^{2}+M^{2} .
$$

The determinant (6) is a generalization of its quantum mechanical $(0+1)$ dimensional version (with $\mu=0$ ) which has been calculated using Green functions [16] and the zeta 
function method [17. Here, we are going to calculate this determinant also using the generalized zeta function method through the basic formula [18]

$$
\operatorname{det} \mathcal{A}=\left.\exp \left\{-\frac{\partial}{\partial s} \zeta(s ; \mathcal{A})\right\}\right|_{s=0}
$$

where the generalized zeta function is defined by $\zeta(s ; A)=\operatorname{Tr} A^{-s}$ and an analytical continuation of $\zeta(s ; A)$ to the whole complex plane of $s$ is tacitly assumed.

For the case at hand, with $\mathcal{A}=-D^{2}+M^{2}$ under $\theta$-periodic boundary conditions, the eingenvalues being given by eq. (7), the generalized zeta function reads

$$
\zeta(s ; \mathcal{A})=\frac{V a_{N}}{(2 \pi)^{N}} \sum_{n=-\infty}^{+\infty} \int_{0}^{\infty} d k k^{N-1}\left(\left[\frac{2 n \pi}{\beta}+i \mu+\frac{\theta}{\beta}\right]^{2}+\vec{k}^{2}+M^{2}\right)^{-s},
$$

where $V$ is the volume, $a_{N}$ is the area of the unit hypersphere, both in $N$ space dimensions. The integral is expressible in terms of the Beta function, and we obtain

$$
\zeta(s ; \mathcal{A})=\frac{C_{N}}{2} \frac{\Gamma\left(\frac{N}{2}\right) \Gamma\left(s-\frac{N}{2}\right)}{\Gamma(s)}\left(\frac{2 \pi}{\beta}\right)^{-2 s+N} \sum_{n=-\infty}^{+\infty}\left[\nu^{2}+(n+\chi)^{2}\right]^{-\left(s-\frac{N}{2}\right)},
$$

where we have defined

$$
C_{N}=\frac{V a_{N}}{(2 \pi)^{N}} ; \quad \nu=\frac{\beta M}{2 \pi} ; \quad \chi=\frac{i \beta \mu+\theta}{2 \pi} .
$$

The sum appearing in eq. (10), well defined for $\Re(s)>1+\frac{N}{2}$, is a generalization of the usual Epstein function [19] but as one can see from formula (8), that the region of interest contains the point $s=0$. The analytic continuation of this sum for the whole complex s-plane can be written as 20]:

$$
\begin{aligned}
& \sum_{n=-\infty}^{+\infty}\left[\nu^{2}+(n+\chi)^{2}\right]^{-\left(s-\frac{N}{2}\right)} \\
= & \frac{\sqrt{\pi}}{\Gamma\left(s-\frac{N}{2}\right)}\left[\frac{\Gamma\left(s-\frac{N}{2}-\frac{1}{2}\right)}{\nu^{2 s-N-1}}+4 \sum_{n=1}^{+\infty} \cos (2 \pi n \chi) \cdot\left(\frac{n \pi}{\nu}\right)^{s-\frac{N}{2}-\frac{1}{2}} K_{s-\frac{N}{2}-\frac{1}{2}}(2 \pi n \nu)\right],
\end{aligned}
$$

where $K_{\alpha}(z)$ is the modified Bessel function of order $\alpha$. Taking the derivative of $\zeta(s, \mathcal{A})$ with respect to $s$, using that $\lim _{s \rightarrow 0}[1 / \Gamma(s)]=0$ and $\lim _{s \rightarrow 0}[(d / d s) \Gamma(s)]=1$ we have, apart from an irrelevant term which is linear in $\beta$ and independent of $\mu$ :

$$
\left.\frac{\partial}{\partial s} \zeta(s, \mathcal{A})\right|_{s=0}=2 \sqrt{\pi} C_{N} \Gamma\left(\frac{N}{2}\right)\left(\frac{2 \pi}{\beta}\right)^{N} \sum_{n=1}^{+\infty} \cos (2 \pi n \chi)\left(\frac{n \pi}{\nu}\right)^{-\frac{1}{2}(N+1)} K_{-\frac{1}{2}(N+1)}(2 \pi n \nu) .
$$


So, using the fact that $\ln \operatorname{det} \mathcal{A}=-\left.(\partial / \partial s) \zeta(s, \mathcal{A})\right|_{s=0}$, we can find the generalized determinant (6) which interpolates continuously the partition functions, or equivalently the free energy, eq. (11), for relativistic boson and fermion gases with chemical potential $\mu$ in $(N+1)$-dimensions:

$$
\begin{aligned}
\Omega(\beta, \mu) & \equiv-\frac{1}{\beta} \ln \mathcal{Z}=-\frac{1}{\beta} \sigma \ln \operatorname{det} \mathcal{A} \\
& =\sigma \frac{V a_{N}}{(2 \pi)^{N}} \frac{1}{\sqrt{\pi}}\left(\frac{2 M}{\beta}\right)^{\frac{1}{2}(N+1)} \Gamma\left(\frac{N}{2}\right) \\
& \times \sum_{n=1}^{+\infty} \cos (n \theta) \cosh (n \beta \mu)\left(\frac{1}{n}\right)^{\frac{1}{2}(N+1)} K_{\frac{1}{2}(N+1)}(n \beta M),
\end{aligned}
$$

where $C_{N}, \nu$ and $\chi$ have been taken from eq. (11). Note that in the above formula, only the real part of the free energy $\Omega(\beta, \mu)$ was taken into account, according to the prescription of introducing the chemical potential as an imaginary time-component gauge potential [10]. If we particularize the parameters $\sigma$ and $\theta$ to the bosonic $(\sigma=-1$ and $\theta=0)$ and fermionic $(\sigma=+1$ and $\theta=\pi)$ cases we shall find precisely the results known in the literature [21]- 23].

To show the equivalence of this partition function with the conventional theory of nonrelativistic anyons, we first recall the cluster expansion

$$
\frac{1}{V} \ln \mathcal{Z}=\sum_{l} b_{l}(V, T) z^{l}
$$

where $z \equiv \exp \beta \mu$ is the fugacity and $b_{l}(V, T)$ are the usually called cluster coefficients. As $\mathcal{Z}=\exp (-\beta \Omega)$, we substitute the expression of $\Omega(\beta, \mu)$, eq. (14), into the above equation and find that the cluster coefficients, in our case, are given by

$$
b_{ \pm n}(V, T)=-2 \sigma\left(\frac{M}{2 \pi n}\right)^{\frac{N+1}{2}} \beta^{\frac{1-N}{2}} \cos (n \theta) K_{\frac{N+1}{2}}(n \beta M) ; \quad(n=1,2,3, \ldots)
$$

The \pm sign for the cluster coefficients are related to particles and antiparticles. Note that, in a conventional nonrelativistic system only particles (or antiparticles) are present, so in that case we would have found only one set of natural cluster coefficients. As they are in fact equal, as required by particle-antiparticle symmetry, this will not modify our analysis.

Now, using the standard formula which relates the second virial coefficient with the cluster ones [24],

$$
B(T)=-\frac{b_{2}(V, T)}{\left[b_{1}(V, T)\right]^{2}},
$$


we have in $N$ space dimensions the exact expression for the relativistic second virial coefficient interpolating the bosonic and fermionic cases:

$$
B(T)=\frac{1}{2 \sigma}\left(\frac{\pi}{M}\right)^{\frac{N+1}{2}} \beta^{\frac{N-1}{2}}\left(1-\tan ^{2} \theta\right) \frac{K_{\frac{N+1}{2}}(2 \beta M)}{\left[K_{\frac{N+1}{2}}(\beta M)\right]^{2}} .
$$

To write the ratio of the two Bessel functions in a more familiar form we can use the asymptotic expression for them

$$
K_{\nu}(x)=\sqrt{\frac{\pi}{2 x}} e^{-x}\left[1+\frac{4 \nu^{2}-1}{8 x}+\frac{\left(4 \nu^{2}-1\right)\left(4 \nu^{2}-3^{2}\right)}{2 !(8 x)^{2}}+\ldots\right]
$$

valid when $-\pi / 2<\arg x<\pi / 2$. Once we are interested in relating our relativistic partition function to the conventional formulation of nonrelativistic anyons, we must reduce our formula by taking the low temperature (nonrelativistic) limit $\beta M>>1$ on equation (18) of the second virial coefficient. In this case, the above asymptotic expression for the Bessel functions can be greatly simplified to

$$
K_{\nu}(x) \simeq \sqrt{\frac{\pi}{2 x}} e^{-x} ; \quad(x>>1),
$$

Form now on, we will restrict our analysis to the particular case of interest, which is $N=2$ space dimensions where anyons live. So considering the low temperature approximation and using eq. (20), we find that eq. (18) reduces to $(\hbar=c=1)$ :

$$
B(T)=\frac{1}{2 \sigma} \frac{\pi \beta}{M}\left(1-\tan ^{2} \theta\right) ; \quad(\beta M>>1) .
$$

We show now that this is equivalent to the results of Arovas et. al. for the second virial coefficient. If one starts from charged fermions interacting with a magnetic flux tube with intensity $\phi=\alpha h c / e$, one gets [8]

$$
B(T)=\frac{1}{4} \lambda_{T}^{2}\left(1-2 \delta^{2}\right)
$$

or analogously, starting from bosons the result is

$$
B(T)=-\frac{1}{4} \lambda_{T}^{2}\left(1-4|\delta|+2 \delta^{2}\right)
$$

where $\lambda_{T}^{2}=\frac{2 \pi \hbar^{2}}{M k T}$ is the thermal wavelength and $\delta$ is the non-integer part of the statistical parameter $\alpha$, i. e., $\alpha=2 j+1+\delta$ for fermions, eq. (22), and $\alpha=2 j+\delta$ for bosons, eq. (23), $j=0, \pm 1, \pm 2, \ldots$ in both cases. 
To compare these results, we note that, in our case, if we start from fermions we should put $\sigma=+1$, identify $\tan ^{2} \theta=2 \delta^{2}$ and get the same answer for the second virial coefficient of anyons, as in eq. (22). Note that these fermions came about through the choice $\theta=\pi$, but we can redefine $\theta=\theta^{\prime}+n \pi$ such that $-\pi / 2 \leq \theta^{\prime} \leq+\pi / 2$, since the $\theta$ dependence appears only in the second virial coefficient through $\tan ^{2} \theta$. In fact, if we want to interpolate the virial coefficients of bosons $\left(-\lambda_{T}^{2} / 4\right)$ and fermions $\left(+\lambda_{T}^{2} / 4\right)$ we must restrict $\tan ^{2} \theta \leq 2$. This restriction, which can be written as $-\arctan \sqrt{2} \leq \theta^{\prime} \leq$ $\arctan \sqrt{2}$, is exactly the one which is needed to map our second virial coefficient onto the one of Arovas et. al.

In the other case, if we start with bosons, we put $\sigma=-1$ and identify $\tan ^{2} \theta=$ $2|\delta|(2-|\delta|)$, in a complete analogous situation where the same restrictions on $\theta$, from the fermionic case, applies here as well. One should also note that, the Arovas et. al. second virial coefficient presents cusp singularities for $\Delta \alpha=2$, which are not present in our result since the coefficient we found is analytical in its parameters except for poles at $\theta=(n+1 / 2) \pi$, where $n=0, \pm 1, \pm 2, \ldots$ This difference comes from the fact that our result also contains values for $B(T)$ which extrapolate the interval $\left(-\lambda_{T}^{2} / 4,+\lambda_{T}^{2} / 4\right)$.

A simpler situation comes up if we consider the particular cases of small angles $\theta \simeq 0$ or $\theta \simeq \pi$, so that

$$
\tan ^{2} \theta \simeq \theta^{2}
$$

In these situations the relation between the quasiperiodic $\theta$ and the statistical parameter $\alpha$ (or $\delta$ ) becomes simply (considering the fermionic anyon model given by (22)):

$$
\sqrt{2} \alpha=\theta \text {. }
$$

So, in the region where anyons are close to fermions (or bosons), where usually perturbation calculations are done for other quantities like the higher virial coefficients, $\theta$ plays the role of the statistical parameter $\alpha$ up to a constant factor $\sqrt{2}$. Of course, the exact relation between these parameters is nonlinear as found above, but in that case the analysis is much more involved and we do not have until now a deeper understanding of this nonlinear behavior.

To understand how we succeed in describing the statistical mechanics of anyons in this simple way, we may say that, the interpolating parameter $\theta$ discussed here plays the role of a topological constant gauge field $A_{\mu}=\left(A_{0}, \overrightarrow{0}\right)$, since the $\theta$-boundary condition for the Klein-Gordon operator, both in the bosonic as well as in the fermionic case, implied that the Matsubara frequencies change according to $\omega_{n} \rightarrow \omega_{n}+\theta / \beta$, which can be viewed as a shift in the time derivative operator, $\partial_{0} \rightarrow \partial_{0}+i \theta / \beta$. As the time coordinate, $x_{0}$, is compactified to the interval $(0, \beta)$, this introduces the non-trivial topology usually associated with anyons but in a different and unexpected manner. This prescription, which works only for the finite temperature case, allows us to relate directly the quasiperiodic boundary condition with intermediate statistics, or anyon description. 
Another interesting point in our formulation is that we can interpolate the bosonic and fermionic second virial coefficients, from two different points of view. First, keeping $\sigma$ fixed to \pm 1 and varying $\theta$ we were able to describe this interpolation very close to the anyon description starting from bosons and fermions with their original equations of motion, or equivalently the bosonic and fermionic determinants, with the inclusion of a topological term, in our case the $\theta$-boundary condition, as we described above. Alternatively, we can keep $\theta$ fixed to 0 or $\pi$ and vary continuously $\sigma$ between +1 and -1 , so that we should identify $\sigma^{-1}=1-2 \delta^{2}$ in the fermionic case and $\sigma^{-1}=1-2|\delta|(2-|\delta|)$ in the bosonic case. This seems to be more close to a q-deformed calculation but this connection is, at least for the moment, far from being trivial.

We can go even further in the determination of the relativistic anyon second virial coefficient, since we do not need to use the low temperature approximation in our calculations. This is so because, in our exact result, eq. (18), we can also use (in $N=2$ ) the exact expression for the Bessel function of order $3 / 2$ :

$$
K_{\frac{3}{2}}(x)=\sqrt{\frac{\pi}{2 x}} e^{-x}\left(1+\frac{1}{x}\right) .
$$

Substituting this expression in the second virial coefficient, eq. (18), instead of the approximate one, eq. (20), we find the exact relativistic result for the anyon second virial coefficient:

$$
B(T)=\frac{1}{2 \sigma} \frac{\pi \beta}{M}\left(1-\tan ^{2} \theta\right)\left(1+\frac{1}{2 \beta M}\right)\left(1+\frac{1}{\beta M}\right)^{-2} .
$$

Note that the possibility of finding this exact result for the second virial coefficient is a peculiarity of odd-dimensional space-times, since for these cases the relevant Bessel functions are of half-integer order for which the asymptotic expansion became exact (see eq. (19)). Naturally, the low temperature result, eq. (21), can be easily obtained from (25) just taking the low temperature limit $\beta M>>1$.

Furthermore, we can also find the high temperature limit (extremely relativistic case) of expression (25) taking $\beta M<<1$ so that we find

$$
B(T)=\frac{1}{4 \sigma} \pi \beta^{2}\left(1-\tan ^{2} \theta\right) ; \quad(\beta M<<1) .
$$

Note that the statistical correction $\left(1-\tan ^{2} \theta\right)$ is independent of the high/low temperature regime, but the mass dependence disappears in the high temperature case, while the power of temperature just changes from $\beta$ to $\beta^{2}$.

As a final comment let us mention that it seems interesting to investigate the extension of these results to other related situations. One of these is the calculation of the higher virial coefficients which are very promising since they can be exactly evaluated in our 
approach [14]. Another situation of interest is the inclusion of an external magnetic field since in most applications of anyonic physics, like the fractional quantum Hall effect, strong external fields are present. We should report on this elsewhere.

Acknowledgments. We thank M. Plyushchay for interesting discussions on relativistic $2+1$ dimensional equations of motion, K. Kirsten for calling our attention to refs. [20, where appear the analytic continuation we needed for the calculation of our generalized zeta function and G. A. Goldin for interesting comments on the manuscript and for calling our attention to refs. «4. H.B.-F. acknowledges Professor R. Jackiw for calling our attention that nonrelativistic anyons at zero temperature obey a spatial quasiperiodic boundary condition [13], for a careful reading of the manuscript, many useful suggestions and his hospitality at the Center for Theoretical Physics - MIT. The authors H.B.-F. and C.F. were partially supported by CNPq (Brazilian agency).

\section{References}

[1] For a review see F. Wilczek (ed.), Fractional Statistics and Anyon Superconductivity, World Scientific, Singapore, 1990.

[2] S. Deser, R. Jackiw and S. Templeton, Phys. Rev. Lett. 48 (1982) 975; Annals of Phys. 140 (1982) 372.

[3] J.H. Leinaas and J. Myrhein, Il Nuovo Cimento 50 (1977) 1.

[4] G. A. Goldin, R. Menikoff and D. H. Sharp, J. Math. Phys. 21 (1980) 650; 22 (1981) 1664; Phys. Rev. Lett. 51 (1983) 2246; G. A. Goldin and D. H. Sharp, Phys. Rev D 28 (1983) 830.

[5] F. Wilczek, Phys. Rev. Lett. 48 (1982) 1144; 49 (1982) 9557.

[6] A. Lerda and S. Sciuto, Nucl. Phys. B 401 (1993) 613.

[7] J. Frolich and P.A. Marchetti, Lett. Math. Phys. 16 (1988) 347.

[8] D. P. Arovas, R. Schrieffer, F. Wilczek and A. Zee, Nucl. Phys. B 251 (1985) 117; see also J. Dowker, J. Phys. A 18 (1985) 3521.

[9] C. Bernard, Phys. Rev. D 9 (1974) 3312.

[10] J. I. Kapusta, Phys. Rev. D 24 (1981) 426; for a review see J. I. Kapusta, Finite temperature field theory, Cambridge, UK, 1989. 
[11] T. Matsubara, Prog. Theor. Phys. 14 (1955) 351.

[12] R. Jackiw and V. P. Nair, Physical Review D 43 (1991) 1933; see also J. L. Cortes and M. S. Plyushchay, Int. J. Mod. Phys. A 11 (1996) 3331.

[13] R. Jackiw, Annals of Physics 201 (1990) 83.

[14] P. Borges, H. Boschi-Filho and C. Farina, in preparation.

[15] F. D. M. Haldane, Phys. Rev. Lett. 67 (1991) 937.

[16] H. Boschi-Filho, C. Farina and A. de Souza Dutra, J. Phys. A 28(1995)L7.

[17] H. Boschi-Filho and C. Farina, Phys. Lett. A 205 (1995) 255.

[18] A. Salam and J. Strahdee, Nucl. Phys. B 90 (1975) 203; J.S. Dowker and R. Critchley, Phys. Rev. D 13 (1976) 3224; S.W. Hawking, Commum. Math. Phys. 55 (1977) 133; G.W.Gibbons, Phys. Lett. A 60 (1977) 385.

[19] P. Epstein, Math. Ann. 56 (1903) 615; 65 (1907) 205.

[20] K. Kirsten, J. Phys. A 25 (1992) 6297; 26 (1993) 2421.

[21] H. E. Haber and H. A. Weldon, Phys. Rev. Lett. 46 (1981) 1497.

[22] H.-T. Elze, W. Greiner and J. Rafelski, J. Phys. A 6 (1980) L149.

[23] A. Actor, Phys. Rev. D 27 (1983) 2548; Ann. of Phys. (N.Y.) 159 (1985) 445; Phys. Lett. B 157(1985)53; Nucl. Phys. B 256 (1986) 689.

[24] J. G. Dash, Films on solid surfaces, Academic Press, N.Y., 1975. 